Tropical Journal of Pharmaceutical Research March 2014; 13 (3): 377-381

ISSN: $1596-5996$ (print); 1596-9827 (electronic)

(C) Pharmacotherapy Group, Faculty of Pharmacy, University of Benin, Benin City, 300001 Nigeria.

All rights reserved.

Available online at http://www.tjpr.org

Original Research Article

http://dx.doi.org/10.4314/tjpr.v13i3.10

\title{
Association of Fibroblast Growth Factor (Fgf-21) as a Screening Biomarker for Chronic Progressive External Ophthalmoplesia
}

\author{
Farnaz Farzaneh Dehkordi ${ }^{1^{*}}$, Massoud Houshmand ${ }^{2}$, Majid Sadeghizadeh ${ }^{3}$, \\ Mohsen Bahmani Kashkouli ${ }^{4}$ and Gholamreza Javadi ${ }^{1}$ \\ ${ }^{1}$ Biology Department, Science and Research Branch, Islamic Azad University, ${ }^{2}$ Medical Genetics Department, National Institute \\ of Genetic Engineering and Biotechnology, ${ }^{3}$ Department of Genetics, Faculty of Biological Sciences, Tarbiat Modares \\ University, ${ }^{4}$ Iran University of Medical Sciences, Tehran, Iran.
}

*For correspondence: Email: farzaneh_farnaz2007@yahoo.com; Tel: +98-9127336690

Received: 15 December 2013

Revised accepted: 19 January 2014

\begin{abstract}
Purpose: To investigate whether or not fibroblast growth factor (FGF-21) can be used as a screening biomarker in chronic progressive external ophthalmoplesia (CPEO) patients.

Methods: FGF-21 concentration was measured in the serum of 24 patients with CEPO phenotype and 24 control samples by enzyme-linked immunosorbent assay (ELISA) and determined the deletion of mitochondrial genome by multiplex polymerase chain reaction (PCR).

Results: FGF-21 concentration in $50 \%$ of CPEO patients showed notable differences from that in control subjects. FGF-21 concentration ratio in patient group, 2 disorder control groups (mitochondrial and non-mitochondrial) and normal group, respectively, was $294.87 \pm 42.10(p<0.0001), 761.78 \pm$ $75.07(p<0.0001), 124.26 \pm 12.27(p=0.1203), 69.27 \pm 10.09(p=0.2195)$. A statistically significant inverse correlation between FGF-21 concentration and age onset was found, with a significant difference $(p<0.05)$ in the age group $\leq 19$ years (mean FGF-21 concentration, $460.36 \mathrm{pg} / \mathrm{mL}$ ) and for the age group $\geq 51$ years (mean concentration FGF-21, $57.87 \mathrm{pg} / \mathrm{mL}$. Surprisingly, there was no significant difference between FGF-21 concentration and age in the mid-age group (20 - 50 years) .

Conclusion: These findings indicate that FGF-21 concentration significantly increases in CPEO patients like in other mitochondrial disorders and this factor can be used as a biomarker in primary diagnosis of mitochondrial disorders. In this regard, FGF-21 assay is only valid in teenagers and the > 50 years age group who show acute symptoms.
\end{abstract}

Keywords: Chronic progressive external ophthalmoplesia, Fibroblast growth factor-21, Mitochondrial disorders, Ophthalmoplesia, Biomarker.

Tropical Journal of Pharmaceutical Research is indexed by Science Citation Index (SciSearch), Scopus, International Pharmaceutical Abstract, Chemical Abstracts, Embase, Index Copernicus, EBSCO, African Index Medicus, JournalSeek, Journal Citation Reports/Science Edition, Directory of Open Access Journals (DOAJ), African Journal Online, Bioline International, Open-J-Gate and Pharmacy Abstracts

\section{INTRODUCTION}

Mitochondrial diseases include a group of metabolic disorders that affect people of all ages [1]. Based on subsarcolemmal accumulations of abnormal mitochondria and their intense red appearance with histological staining, mitochondrial diseases are divided in two major groups; ragged-red fiber disorders and non- ragged-red fibers ones. The first group includes diseases such as Kearns-Sayre syndrome (KSS), mitochondrial encephalopathy with lactic acidosis and stroke-like episodes (MELAS), myoclonic epilepsy with ragged red fibers (MERRF), Pearson syndrome, progressive external ophthalmoplegia (PEO) $[2,3]$. 
CPEO is a mitochondrial myopathy that causes muscular or multisystem symptoms [4]. The disease is characterized by a progressive involvement of the ocular musculature, with ptosis of the eyelids and impairment of eye movements $[4,5]$. Lactate, pyruvate, aminoacids and creatine kinase are considered as serum markers for the diagnosis of mitochondrial disorders. However, since they do not have a good sensitivity and specificity for such a detection muscle biopsy is considered as a better tool for detecting extensive intra-muscular mitochondrial damages [6-8]. On the other hand, biopsy is an invasive procedure that is more costly than testing a serum biomarker; furthermore, children would require a general anesthesia in biopsy.

Suomalainen et al introduced fibroblast growth factor 21 (FGF-21) as a new serum biomarker in patients with mitochondrial dysfunctions [7]. Consequently, the aim of this study was to determine if the measurement of FGF-21 concentration in the serum of Iranian patients with CPEO can be used as a biomarker for human mitochondrial disorder.

\section{EXPERIMENTAL}

\section{Patients}

24 patients (14 men and 10 women) with CPEO were referred to the Genetics Department at Special Medical Centre (SMC) of Tehran for diagnosis. Documented, written consent was obtained from all patients who agreed to participate. The study protocol was approved by SMC project Nr 385 ethics committee according to World Medical Association's Declaration of Helsinki [9]. Our study was performed according to WHO criteria [10]. Serum samples were collected from CPEO patients and control groups, comprised of two groups of nonmitochondrial (control group 1) and other mitochondrial disorders except CPEO (control group 2), e.g., mitochondrial myopathy, Leber's hereditary optic neuropathy (LHON), mitochondrial encephalomyopathy, lactic acidosis, and stroke (MELAS); control group 2 consisted of 9 mitochondrial and 5 nonmitochondrial disorder patients, while the healthy control group comprised of 5 men and 5 women. These cases are listed in Table 1 and Table 2.

\section{FGF-21 concentration measurement}

ELISA (BioVendor Human FGF-21 ELISA Kit) was used to measure FGF-21 concentration in serum samples. For FGF-21, the cutoff was based on the reference ranges stated in the study of the Suomalainen et al. [7]. Thus, the cutoff value used ws $200 \mathrm{pg} / \mathrm{mL}$.

\section{Molecular study}

Due to the fact that in most cases, CPEO occurs as result of a sporadic deletion of mitochondrial genome, multiplex polymerase chain reaction (PCR) was used to study mtDNA in patients who showed symptoms of the disease. Six primers were used to analyze the whole mitochondrial genome.

A pair of primers related to the mtDNA region that is generally not deleted was used as internal control, and the others were used to show delete regions. The PCR of each sample was set in a $0.2 \mathrm{ml}$ tube using $100 \mathrm{mg}$ of total DNA, $10 \mathrm{pmol}$ of each primer, $200 \mathrm{mmol}$ of dNTPs, 1X PCR buffer containing $2.5 \mathrm{mmol} \mathrm{MgCl} 2$ and $1 \mathrm{U}$ Taq DNA polymerase (Roche Diagnostics, Mannheim, Germany). The cycling conditions used for the amplification were as follows: initial denaturation at $94{ }^{\circ} \mathrm{C}$ for $5 \mathrm{~min}$ followed by 35 cycles of denaturation for $1 \mathrm{~min}$ at $94{ }^{\circ} \mathrm{C}$, annealing for $1 \mathrm{~min}$ at $55^{\circ} \mathrm{C}$ and extension for 35 $\mathrm{sec}$ at $72^{\circ} \mathrm{C}$ (final extension for $10 \mathrm{~min}$ ).

\section{Statistical analysis}

$P<0.05$ was considered statistically significant and this was assessed using Duncan's multiple range test to compare FGF-21 concentrations between patients and controls. Also compared were FGF-21 concentrations between the 3 age groups ( $\leq 19$ years $20-50$ years and $\geq 51$ years) in CPEO patients. Data are expressed as mean \pm standard error (SE).

\section{RESULTS}

The concentration of FGF-21 in $50 \%$ of cases (total: 48) with CPEO phenotype was not in the normal range. While there was no increase in FGF-21 concentration in healthy and nonmitochondrial disorder control groups, a higher concentration was detected in the mitochondrial disorder groups (CPEO and other mitochondrial disorders). The outcomes of statistical comparisons of the pertinent parameters are shown in Tables 3 and 4.

Genetic analysis showed that $50 \%$ of patients with CPEO clinical symptoms as well as increased serum FGF-21 concentration, there was deletion of mtDNA, while in the other patients with CPEO clinical symptoms and normal serum FGF-21 concentration, there was no deletion of mtDNA. The observed bands on 
Table 2: Serum FGF-21 concentrations in 3 control groups; disease control group (non-mitochondrial: mental retardation, epilepsy, dismorphia, DMD and other mitochondrial disorders: LHON, MELAS, mitochondrial myopathy) and healthy control group.

\begin{tabular}{|c|c|c|c|c|}
\hline Case no. & Sex & Age (years) & Clinical feature & FGF-21 $(\mathrm{pg} / \mathrm{ml})$ \\
\hline 1 & $\mathrm{~F}$ & 44 & LHON & 789.8 \\
\hline 2 & $\mathrm{~F}$ & 52 & LHON & 1549 \\
\hline 3 & M & 17 & LHON & 451.6 \\
\hline 4 & M & 22 & LHON & 824.0 \\
\hline 5 & $\mathrm{~F}$ & 20 & MELAS & 723.1 \\
\hline 6 & M & 51 & MELAS & 612.8 \\
\hline 7 & M & 55 & Mitochondrial myopathy & 619.0 \\
\hline 8 & $\mathrm{~F}$ & 27 & Mitochondrial myopathy & 830.0 \\
\hline 9 & $\mathrm{~F}$ & 36 & Mitochondrial myopathy & 456.8 \\
\hline 10 & $\mathrm{~F}$ & 28 & Mental retardation & 62.4 \\
\hline 11 & $\mathrm{~F}$ & 18 & Epilepsy & 169.1 \\
\hline 12 & $\mathrm{~F}$ & 22 & Epilepsy & 151.3 \\
\hline 13 & M & 38 & Dismorphia & 128.2 \\
\hline 14 & M & 29 & DMD & 110.3 \\
\hline 15 & $\mathrm{~F}$ & 15 & Healthy & 48.2 \\
\hline 16 & $\mathrm{~F}$ & 43 & Healthy & 72.7 \\
\hline 17 & $\mathrm{~F}$ & 23 & Healthy & 24.4 \\
\hline 18 & M & 45 & Healthy & 16.7 \\
\hline 19 & $\mathrm{~F}$ & 56 & Healthy & 112.5 \\
\hline 20 & M & 47 & Healthy & 177.0 \\
\hline 21 & $M$ & 52 & Healthy & 56.7 \\
\hline 22 & $\mathrm{~F}$ & 32 & Healthy & 48.3 \\
\hline 23 & $M$ & 26 & Healthy & 72.9 \\
\hline 24 & $\mathrm{M}$ & 34 & Healthy & 63.3 \\
\hline
\end{tabular}

Table 3: Statistical comparison of FGF-21 factor between 4 groups; (group 1: CPEO patients; group 2: other mitochondrial disorders; group 3: non-mitochondrial disorders; and group 4: healthy control group)

\begin{tabular}{llllll}
\hline Group & Minimum concentration & Maximum concentration & Mean \pm SE & Duncan grouping & $P$-value \\
\hline 1 & 8.20 & 849.80 & $294.87 \pm 42.10$ & B & $<.0001$ \\
2 & 451.60 & 1549.00 & $761.78 \pm 75.07$ & A & $<.0001$ \\
3 & 62.40 & 169.10 & $124.26 \pm 12.27$ & $\mathrm{C}$ & 0.1203 \\
4 & 16.70 & 177.00 & $69.27 \pm 10.09$ & $\mathrm{C}$ & 0.2195 \\
\hline
\end{tabular}

Table 4: Statistical comparison of FGF-21 factor between 3 age groups; (group 1: $\leq 19$ years old; group 2: 20 50 years old; and group 3: $\geq 51$ years old).

\begin{tabular}{llllllll}
\hline Group & Frequency & $\%$ & $\begin{array}{l}\text { Minimum } \\
\text { concentration }\end{array}$ & $\begin{array}{l}\text { Maximum } \\
\text { concentration }\end{array}$ & Mean \pm SE & $\begin{array}{l}\text { Duncan } \\
\text { grouping }\end{array}$ & $P$-value \\
\hline 1 & 6 & 25.00 & 90.40 & 849.80 & $460.36 \pm 115.14$ & A & 0.0005 \\
2 & 14 & 58.33 & 8.20 & 836.40 & $291.66 \pm 81.58$ & A,B & 0.0007 \\
3 & 4 & 16.67 & 42.50 & 88.80 & $57.87 \pm 10.49$ & B & 0.6802 \\
\hline
\end{tabular}




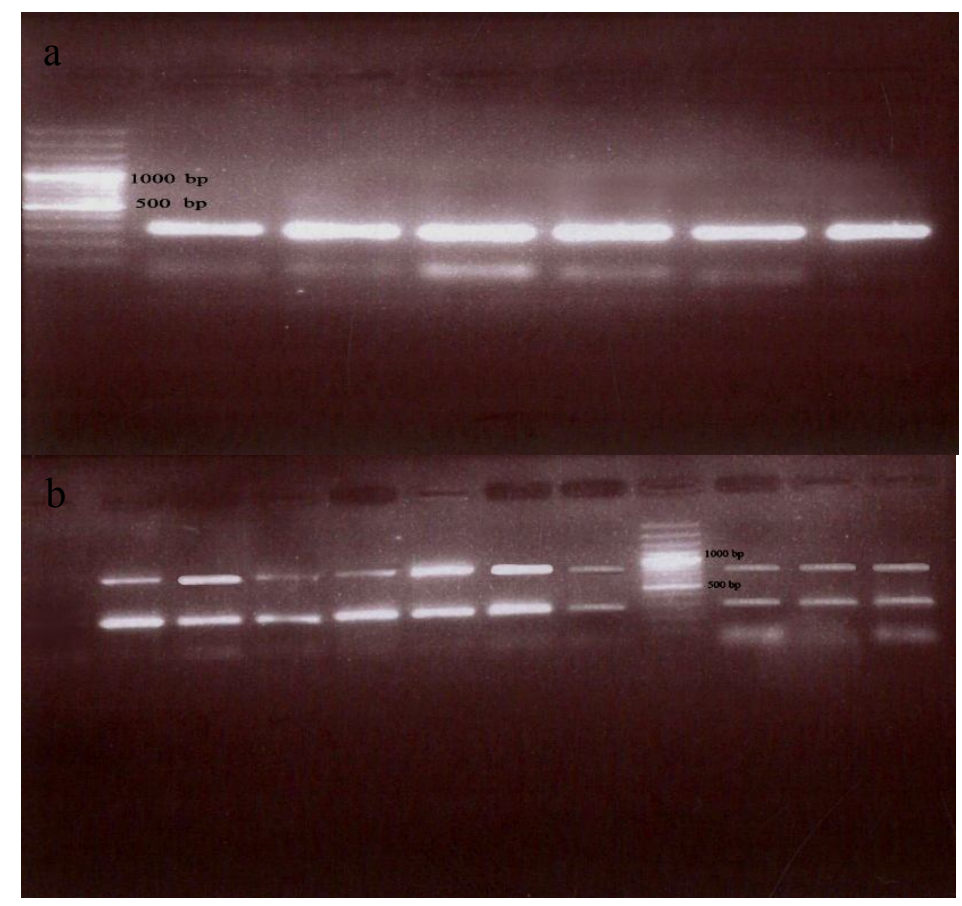

Figure 1: Multiplex PCR using ONP 86, ONP 89 and ONP 10 primers. a = left to right; 5 first are related to CPEO patients without increase in FGF-21 concentration and the other is related to normal individuals. They show only the internal control band in size of $279 \mathrm{bp} ; \mathrm{B}=$ The bands are related to CPEO patients with increase in FGF-21 concentration. They all show 2 bands, $279 \mathrm{bp}$ band is related to ONP 86 and ONP 89 primers as a internal control and the upper band is related to ONP 66 and ONP 10 primers that polymerase the DNA when the deletion was occurred. The size of this band is $870 \mathrm{bp}$.

agarose gel electrophoresis showed the existence of deletion in the region between two primers (Figure 1). The size of the deleted region in the CPEO patients with increase in FGF-21 concentration was $870 \mathrm{bp}$.

\section{DISCUSSION}

Mitochondrial disorders are those types of metabolic diseases that lead to decrease of energy in damaged organs due to defect in respiratory chain complexes. However, brain, kidney, liver and muscles are the organs that use most of the body energy and so are more prone to damage [11]. According to the justification of Suomalainen et.al [7], FGF-21 is the factor that plays an important role in lipid metabolism and also FGF-21 concentration increases in individuals with mitochondrial disease such as MELAS, mitochondrial recessive ataxia syndrome (MIRAS), mitochondrial neurogastrointestinal encephalopathy (MNGIE); the authors proposed this factor as a serum biomarker for the diagnosis of mitochondrial disorders [7,12], due to the diffulty in carrying out eyelid muscular biopsy in CPEO patients with ophtalmoplasia phenotype. This is why the concentration of FGF21 as a biomarker in this type of mitochondrial diseases was undertaken in this study.
The results obtained in this study show that FGF21 could become a suitable general biomarker for the diagnosis of mitochondrial diseases. These findings reveal that FGF-21 concentration will significantly increase in CPEO patients like other mitochondrial disorders and hence this factor can serve as a biomarker in the primary diagnosis of mitochondrial disorders. We also suggest $200 \mathrm{pg} / \mathrm{mL}$ as a cutoff concentration.

Increased concentration of the test factor in $50 \%$ of cases with confirmed CPEO phenotype was observed and that it was not gender-dependent; however, with regard to age groups, significant differences were observed except for the $20-50$ years age group.

Although genetic studies on the patients who showed increased FGF-21 concentration demonstrated partial deletion of mtDNA, similar deletion in the other $50 \%$ of patients who exhibited normal FGF-21 concentration This finding could suggest another genetic factor other than mtDNA deletion in the phenotype of ophtalmoplasic patients, or misdiagnosis of the patients. It must be mentioned that Suomalainen et.al's study was on patients who definitely suffer from mitochondrial disorders. In the present, we had no access to histological data on the eyelids and definite biochemical markers such as COX1 
for diagnosis of mitochondrial disorders in Iran. Nonetheless, this study suggests the possibility of the application of FGF-21 assay for diagnosis of patients who definitely suffer from mitochondrial disorders.

\section{CONCLUSION}

It is postulated that FGF-21 factor could serve as a serum biomarker for the early diagnosis of CPEO in patients with definite mtDNA deletion. The low population rate of this phenotype in Iran would limit a definitive statement on the relationship between the genotype of this disease (deletion or non-deletion of mtDNA) and increased FGF-21 concentration. However, our findings indicate that the application of serum FGF-21 concentration not only eliminates the need for muscular biopsy but also can be used as a predictive test for those CPEO patients who have deletion in mtDNA but could not be distinguished from other similar clinical phenotypes (phenocopies).

\section{ACKNOWLEDGEMENT}

The authors appreciate the support of colleagues in Tehran Special Medical Center, Tehran, Iran during the study, as well as financial and institutional support from the Tehran Special Medical Center.

\section{REFERENCES}

1. Schaefer AM, McFarland R, Blakely EL, He L, Whittaker $R G$, Taylor RW, Chinnery PF, Turnbull DM.
Prevalence of mitochondrial DNA disease in adults. Ann Neurol 2008; 63(1): 35-39.

2. Ellinas H, Frost E. Mitochondrial disorders. Middle East $J$ Anesthesiol 2011; 21(2): 235-242.

3. McFarland R, Turnbull DM. Batteries not included: diagnosis and management of mitochondrial disease. J Intern Med 2009; 265: 210-228.

4. Domenis DR, Okubo PM, Sobreira C, Dantas $R O$. Esophageal Contractions in Patients with Chronic Progressive External Ophthalmoplegia. Dig Dis Sci 2011; 56: 2343-2348.

5. Shimko, Jacqueline W. Chronic Progressive External Ophthalmoplegia. Am Orthopic J 2005; 55: 13-18.

6. Rodenburg RJ. Biochemical diagnosis of mitochondrial disorders. J Inherit Metab Dis 2011; 34: 283-292.

7. Suomalainen $A$, Elo JM, Pietiläinen $K H$, Hakonen $A H$, Sevastianova $K$, Korpela $M$, Isohanni $P$, Marjavaara SK, Tyni T, Kiuru-Enari S, et al. FGF21 as a biomarker for muscle-manifesting mitochondrial respiratory chain defi ciencies: a diagnostic study. Lancet Neurol 2011; 10: 806818.

8. Shaham O, Slate NG, Goldberger $O, X u Q$, Ramanathan $A$, Souza AL, Clish CB, Sims KB, Mootha VK. A plasma signature of human mitochondrial disease revealed through metabolic profi ling of spent media from cultured muscle cells. Proc Natl Acad Sci USA 2010; 107: 1571-1575.

9. World Medical Association. Declaration of Helsinki Ethical Principles For Medical Research Involving Human Subjects. 59th WMA General Assembly, Seoul, October 2008.

10. World Health Organization. International Ethical Guidelines for Biomedical Research Involving Human Subjects. Council for International Organizations of Medical Sciences 2002.

11. Cohen BH, Gold DR. Mitochondrial cytopathy in adults: what we know so far. Cleve Clin J Med 2001; 68(7): 625-626, 629-642.

12. Murata $Y$, Konishi M, Itoh N. FGF21 as an Endocrine Regulator in LipidMetabolism: From Molecular Evolution to Physiology and Pathophysiology. J Nutr Metab 2011; 2011: 1-8. 